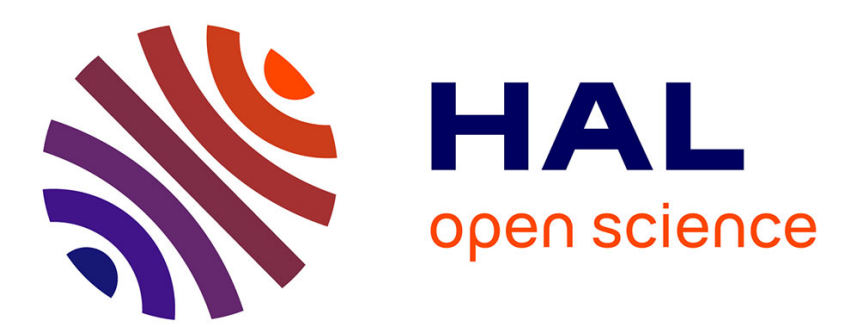

\title{
Combining criteria for the detection of incorrect entries of non-native speech in the context of foreign language learning
}

Luiza Orosanu, Denis Jouvet, Dominique Fohr, Irina Illina, Anne Bonneau

\section{- To cite this version:}

Luiza Orosanu, Denis Jouvet, Dominique Fohr, Irina Illina, Anne Bonneau. Combining criteria for the detection of incorrect entries of non-native speech in the context of foreign language learning. SLT 2012 - 4th IEEE Workshop on Spoken Language Technology, Dec 2012, Miami, United States. hal-00753458

\author{
HAL Id: hal-00753458 \\ https://hal.inria.fr/hal-00753458
}

Submitted on 25 Mar 2016

HAL is a multi-disciplinary open access archive for the deposit and dissemination of scientific research documents, whether they are published or not. The documents may come from teaching and research institutions in France or abroad, or from public or private research centers.
L'archive ouverte pluridisciplinaire HAL, est destinée au dépôt et à la diffusion de documents scientifiques de niveau recherche, publiés ou non, émanant des établissements d'enseignement et de recherche français ou étrangers, des laboratoires publics ou privés. 


\title{
COMBINING CRITERIA FOR THE DETECTION OF INCORRECT ENTRIES OF NON-NATIVE SPEECH IN THE CONTEXT OF FOREIGN LANGUAGE LEARNING
}

\author{
Luiza Orosanu, Denis Jouvet, Dominique Fohr, Irina Illina, Anne Bonneau
}

\author{
Speech Group, LORIA \\ Inria, Villers-les-Nancy, F-54600, France \\ CNRS, LORIA, UMR 7503, Villers-les-Nancy, F-54600, France \\ Universite de Lorraine, LORIA, UMR 7503, Villers-les-Nancy, F-54600, France \\ \{luiza.orosanu, denis.jouvet, dominique.fohr, irina.illina, anne.bonneau\}@loria.fr
}

\begin{abstract}
This article analyzes the detection of incorrect entries of nonnative speech in the context of foreign language learning. The purpose is to detect and reject incorrect entries (i.e. those for which the speech signal does not correspond at all to the associated text) while being tolerant to the mispronunciations of non-native speech. The proposed approach exploits the comparison between two text-to-speech alignments : one constrained by the text which is being checked, with another one unconstrained, corresponding to a phonetic decoding. Several comparison criteria are described and combined via a logistic regression function. The article analyzes the influence of different settings, such as the impact of non-native pronunciation variants, the impact of learning the decision functions on native or on non-native speech, as well as the impact of combining various comparison criteria. The performance evaluations are conducted both on native and on non-native speech.
\end{abstract}

Index Terms - Foreign language learning, incorrect entries, non-native speech, constrained and unconstrained alignments

\section{INTRODUCTION}

Support for foreign language learning is an application area of automatic speech recognition technologies. Their objective is to detect and to provide feedback on pronunciation errors, in order to help correcting them and slowly improve the foreign language proficiency. One of the main difficulties for such a system is to automatically detect and locate pronunciation errors [1], while remaining robust to non-native speech. Several methods have been proposed to determine a score for the pronunciation quality [2], by exploring likelihood ratios. Such systems benefit from the introduction of

The work presented in this article is part of the ALLEGRO project, funded by the European program INTERREG IV. acoustic models of native phonemes (in addition to those belonging to the target language), along with the a priori knowledge of possible non-native mispronunciations.

The prosody is an other important element of foreign language learning. Some projects have addressed the feedback on duration errors [3]. An original method was proposed in [4], which aims to improve both production and perception, by combining an accurate and detailed prosodic feedback with an audio feedback based on a modification of the learner's pronunciation. This approach requires a phonetic segmentation of the learner's utterance; a study of the relevance of the phonetic segmentation has been undertaken in [5]. These automatic methods for pronunciations diagnosis are based on a phonetic segmentation of the speech signal, which is obtained via a forced alignment with the models corresponding to the pronounced sentence. The inclusion of non-native pronunciation variants improves the quality of alignments [6].

However, the learner does not always pronounce (entirely or at all) the sentence requested by the learning exercise (error of pronunciation, speech interference, sound capture issue, ...). Hence, before analyzing the quality of the learner's pronunciation or even working on obtaining a relevant prosodic feedback, the system must be able to determine if the audio signal actually corresponds to the expected sentence. So, the objective of this study is to detect and to reject incorrect entries, while being tolerant to non-native mispronunciations. With this kind of filtering, we can be sure that the data on which we will be working on is acceptable (or maybe even $100 \%$ correct), and thus further detailed processing and analysis will be relevant.

In speech recognition, such an acceptation/rejection task typically corresponds to the detection of out of vocabulary words $[7,8]$ or the detection of sentences that do not contain any keywords [9]. Similar techniques are also used for validating speech corpora (i.e. match between transcription and speech signal) [10]. Unlike these studies, which mainly aim the native speech, here we deal with non-native speech. 
This article performs a detailed study on the rejection of incorrect entries in the context of foreign language learning. The paper analyzes the impact of various comparison criteria, both on native and on non-native speech. The first section provides a description of our methodology, in particular the criteria used to distinguish correct entries from incorrect ones, along with the chosen classifier. The second part of the paper is devoted to the description of experiments and the discussion of results.

\section{METHODOLOGY}

In order to reject incorrect entries, while accepting those that are correct, we must determine whether or not the audio signal corresponds to the expected sentence. For that, a decision was taken : to decode the audio signals in three different ways (one constrained alignment, and two unconstrained decodings) and to compare the resulting phonetic segmentations.

\subsection{Phonetic segmentations}

1. Constrained decoding (forced alignment) : the system is forced to follow the sequence of words within the expected text.

2. Phonetic decoding based on phoneme loop : the system is free to choose any phoneme in any position in the sentence.

3. Phonetic decoding based on word loop : the system is free to choose any word (limited to the $\sim 200$ words of the learning application) in any position in the sentence.

For the segmentations (1) and (3), the words may have several pronunciation variants, native and/or non-native, according to the associated lexicon.

\subsection{Comparison criteria}

The comparison criteria serve to distinguish correct entries from incorrect ones. They are based on information extracted from the constrained and unconstrained segmentations. Two comparisons are made : first one between the segmentations (1) and (2) ("phoneme loop comparison"), and the second one between the segmentations (1) and (3) ("word loop comparison"). The comparison takes into account the phonemes, the frames, the non-speech segments, the likelihood ratios or the phoneme durations.

1. Criterion associated to the phonemes : percentage of phonetic segments that have the same label in both segmentations and where starting or ending temporal boundaries are within a $20 \mathrm{~ms}$ interval. The non-speech segments are ignored. This criterion is generally greater for correct entries than for incorrect ones.
2. Criterion associated to the frames : percentage of frames whose labels belong to the same phonetic class in both segmentations. A phonetic class is represented by sounds which share at least one phonetic feature, and especially the "manner of articulation" (e.g stop class, fricative class, ...). Even if the phonetic decoding does not always find the correct phoneme, it is likely to replace it with another one belonging to the same class. This criterion is generally greater for correct entries than for incorrect ones.

3. Criterion associated to the non-speech segments : duration difference of non-speech segments between the two segmentations (as a percentage of the total duration of the utterance). When the system is forced to align an audio signal on a non-matching text (the case of an incorrect entry), it is likely to add several non-speech segments between words and / or increase or decrease the duration of those that actually exist. This criterion is generally smaller for correct entries than for incorrect ones.

4. Criterion associated to the $\log$ likelihood ratio : difference between the logarithmic likelihoods of both segmentations. A value close to " 0 " indicates that both segmentations lead to the same logarithmic likelihood, which means that they correspond to the same sequence of phonemes (a correct entry). The log likelihood ratio gets smaller (negative value) for incorrect entries.

5. Criterion associated to the phoneme durations : difference between the number of short phonemes (having the minimal duration of exactly 3 frames which corresponds to the 3 emitting states of the HMM, Hidden Markov Model) within both segmentations (as a percentage of the total number of phonemes within the forced alignment). A significant quantity of phonemes with minimal durations could indicate abnormalities within the alignment. This criterion is generally smaller for correct entries than for incorrect ones.

\subsection{Data classification}

Given the comparison criteria (section 2.2) and the classification task limited to two classes (correct or incorrect), the predictive model of the logistic regression [11] was chosen as binary classifier. The logistic regression is used here to compute an entry's probability of being correct :

$$
f(\bar{X})=\frac{1}{1+\exp \left(-\left(\alpha_{0}+\alpha_{1} x_{1}+\ldots+\alpha_{k} x_{k}\right)\right)}
$$

The first step of the approach is to train the parameters of the classifier on the training data. The training data is represented by the data set

$$
D=\left\{\overline{X_{i}}, y_{i}\right\}, i=1, \ldots, N
$$





Fig. 1. Impact of lexicon and training data on DET curves for native data (a) and for non-native data (b), when incorrect entries are represented by entirely modified transcriptions and when using 10 comparison criteria

where:

$-\bar{X}=<x_{1}, x_{2}, \ldots, x_{k}>$ is the vector containing the entry's informations, in other words the $k$ comparison criteria ( $k$ is maximum 10$)$

$-y$ indicates the belonging class: correct 1 , incorrect 0

- $N$ is the number of entries within the training data set.

We train the unknown $\alpha=\left(\alpha_{0}, \alpha_{1}, \ldots, \alpha_{k}\right)$ parameters by minimizing the error function $E$. The error function indicates the mismatch between the class membership (which is either 0 or 1) and the $f(\bar{X})$ value of the logistic function which varies between 0 and 1 .

$$
E=-\sum_{i=1}^{N}\left(y_{i} \cdot \ln \left(f\left(\overline{X_{i}}\right)\right)+\left(1-y_{i}\right) \cdot \ln \left(1-f\left(\overline{X_{i}}\right)\right)\right)
$$

The minimization is performed using the gradient descent method. This numerical algorithm seeks an optimum (possibly local) by successive improvements. From an $\alpha$ starting point, the parameters are continuously modified until a stop condition is reached (improvement smaller than a given threshold).

Then, the DET curves ("detection error trade-off") are used to represent the results. A DET curve is an error-rate graphic for binary classification systems. It is used here to represent the performance on the task of entries classification, which involves a compromise between the rates of "false acceptance" ( $F A$, percentage of incorrect entries classified as correct by the system) and "false rejection" ( $F R$, percentage of correct entries classified as incorrect by the system). An entry is accepted as correct only if the logistic regression's value $f(\bar{X})$ (for the chosen $\bar{X}$ criteria) is greater than a threshold $\sigma$. To plot the DET curve, several values for the threshold $\sigma \epsilon[0,1]$ are considered. The (FA, FR) error rates for each threshold value are marked on the graphic. Finally, the best compromise between the error rates (among all the available points on the DET graphic) is the one that maximizes the following F-measure:

$$
\frac{1}{F}=\frac{1}{2} \cdot\left(\frac{1}{1-F A}+\frac{1}{1-F R}\right)
$$

\section{EXPERIMENTS AND RESULTS}

\subsection{Experimental setup}

To evaluate the approaches mentioned in section 2, two English corpora were used (one native and the other one nonnative). They were both created for the INTONALE project [12], which is devoted to prosodic studies. The native corpora contains approximately 1500 audio signals, recorded by 22 English speakers (15 women and 7 men, 66 sentences per speaker). The non-native corpora contains about 800 audio signals, recorded by 34 French speakers ( 29 women and 5 men, 23 sentences per speaker). The recordings were made in a quiet room. The software developed for the recordings displays a sentence on the screen. The speaker can choose, after each pronunciation of a sentence, to repeat it (in case of problems) or to move on to the next.

All used corpora contain only correct entries (even if the non-native speech is subject to many mispronunciations). To simulate incorrect entries, we used the same audio signals, but we attached to each of them a text that does not correspond to it. We modified the transcripts in two different ways: by replacing a word or a sequence of words (the sequence of words is extended up to reaching a minimal size of 3 syllables, 4 syllables, 5 syllables, 6 syllables or 7 syllables) or by replacing the entire sentence. These replacements between words and between sentences are random. Even though these incorrect entries are artificial, we assume them to be relevant to our task, given that we need to prepare ourself to the outcome where the learner might pronounce anything else but what he 


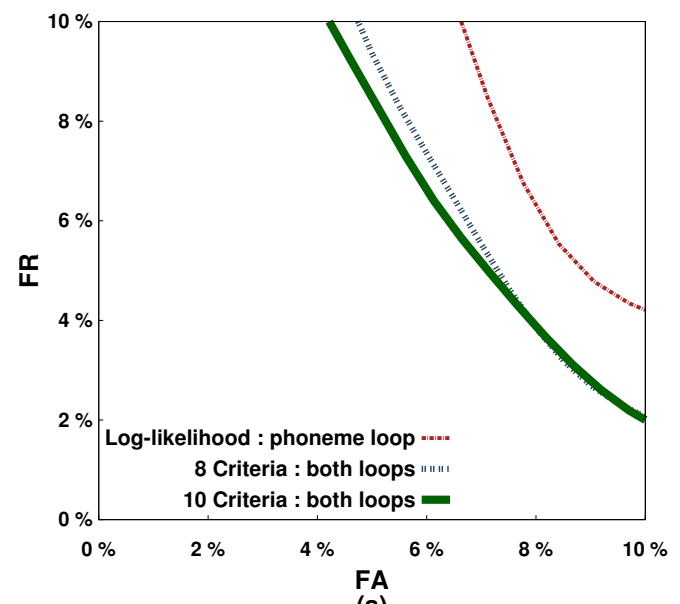

(a)

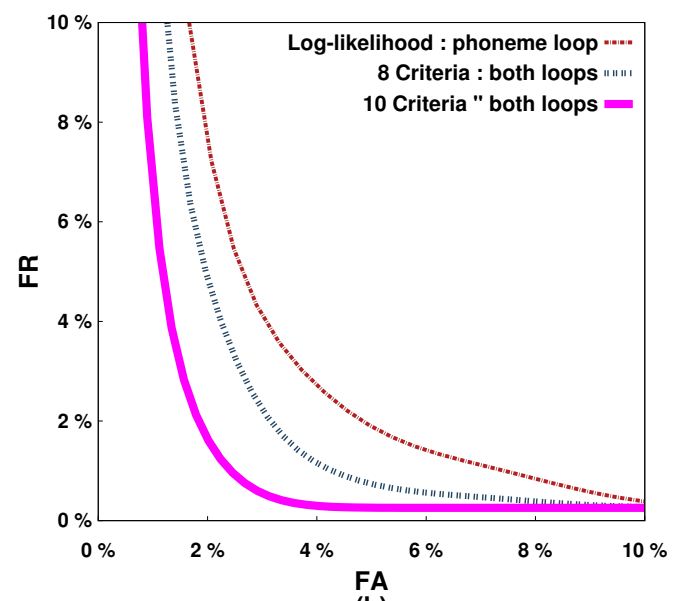

(b)

Fig. 2. Impact of combining various comparison criteria on DET curves for native data (a) and for non-native data (b), when incorrect entries are represented by entirely modified transcriptions and when using native trained parameters and the native lexicon to evaluate native data and when using non-native trained parameters and the non-native lexicon to evaluate non-native data

is asked. Afterwards, each corpora, native or non-native, was divided in two equal parts, one meant to train the parameters, and the other one to evaluate their performance.

The HTK tools [13] were used to decode the audio signals. The MFCC (Mel Frequency Cepstral Coefficients) acoustic analysis gives 12 MFCC parameters and a logarithmic energy per frame (window of $32 \mathrm{~ms}, 10 \mathrm{~ms}$ shift). The forced alignment of an entry is done by using contextindependent HMM acoustic models (42 native-English models, a silence model, a noise model and three native-French models: schwa, /y/, / $\tilde{\mathrm{a}} /$ ) and by taking into account pronunciation variants of each word. Each HMM state has been modeled with a 16 Gaussian mixture. The English acoustic models and the silence model were trained using the TIMIT's corpus [14]. The French acoustic models and the noise model were trained using the ESTER2's corpus [15].

Two lexicons were used. The first one includes only native variants for each word (native lexicon: the CMU dictionary [16]). The second one includes also non-native variants, those which were observed at least two times in the pronunciation of non-native speech (non-native lexicon).

\subsection{Evaluation of the lexicon and the training data set}

This section studies the impact of using a native or a nonnative lexicon, along with the impact of using a native or a non-native data set for training the parameters of the decision function.

Given that it is not possible to know in advance if the pronounced sentence will be entirely or just partially different from the expected sentence, we chose to execute a single and global training of the logistic function, on all our groups of correct \& incorrect data sets ( "3 syllables" = the group of modified transcripts where we replaced a word or a group of words having a minimal size of 3 syllables, ..., "all syllables" $=$ the group of modified transcripts where we replaced the entire sentence). We test it afterwards on each group separately.

Figure 1 presents the impact of the lexicon and of the training data set (note that all curves are smoothened and that their order is indicated in the legend). We report the results obtained on the "all syllables" data sets, using the combination of all 10 comparison criteria. The curves show that it is important to learn the decision functions on the same type of data for optimum results. They also show that the use of nonnative pronunciation variants in the lexicon is necessary for non-native speech. Therefore, the best results are obtained on native data, with the use of a native lexicon and training on native data (the 'native training, native lexicon' curve in graph (a)), and on non-native data, with the use of a non-native lexicon and training on non-native data (the 'non-native training, non-native lexicon' curve in graph (b)).

\subsection{Evaluation of the comparison criteria}

This section studies the impact of combining various comparison criteria.

Figure 2 presents the impact of different criteria combinations. The results obtained with a single criterion ( the 'Loglikelihood: phoneme loop' curve) are improved with the use of the first four criteria $(1,2,3 \& 4$ in section 2.2$)$ computed from both the phoneme loop and the word loop comparisons (the '8 Criteria : both loops' curve). Further improvement is obtained with the use all 10 criteria (the '10 Criteria: both loops' curve). 


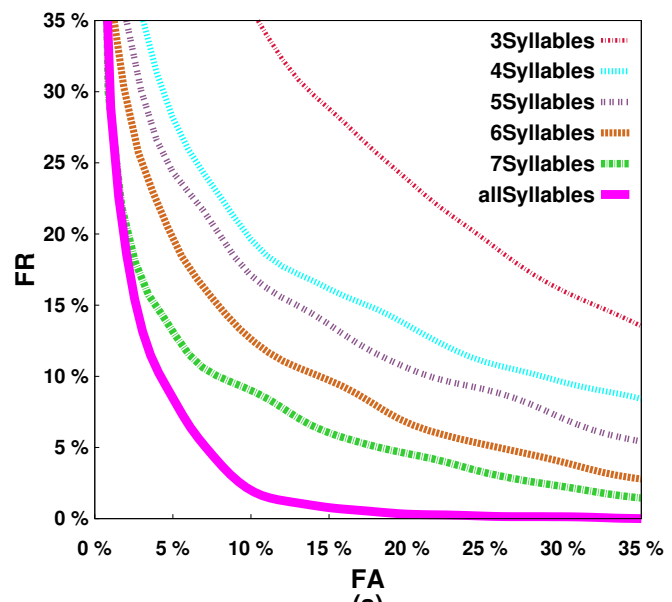

(a)

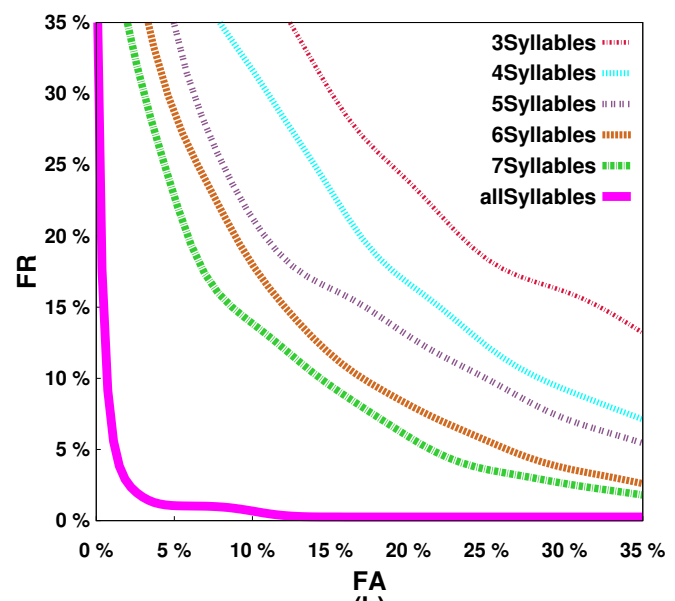

(b)

Fig. 3. Evaluation of DET curves on native data (a) and on non-native data (b), according to the size of the incorrect part of the entry, when using 10 comparison criteria and when using native trained parameters and the native lexicon to evaluate native data and when using non-native trained parameters and the non-native lexicon to evaluate non-native data

\subsection{Evaluation of the performance}

This section studies the performance of our classifier.

Figure 3 presents the results obtained on the different data sets. As expected, the performance increases with the number of differences between the pronounced sentence and the expected sentence.

Table 1 presents some numerical results. The best error rates obtained for native data are $8.01 \%$ (FA) and $3.31 \%$ (FR), corresponding to a F-measure (eq. 3) of about $94 \%$, and for non-native data : $2.06 \%$ (FA) and $0.77 \%$ (FR) corresponding to a F-measure of about $99 \%$.

Figure 4 presents the overall performance of our classifier. We calculate for each modified transcription its distance with respect to the original transcription. This distance indicates the minimal number of changes needed in order to match both sentences (possible changes: insert a phoneme, delete a phoneme, substitute a phoneme). The obtained distances vary between 1 and 51 phoneme changes. The results are grouped with respect to their distances (several intervals of distances are considered) and the graphic displays the corresponding Fmeasures. Starting from a distance of 6 phoneme changes we can obtain a performance greater than $80 \%$.

The results obtained on native data are slightly worse than the results obtained on non-native data; one possible explanation comes from a detailed analysis of the corpora which showed that the native data used in our experiments was pronounced with a higher speaking rate and that the noise level was also higher. The higher speaking rate observed on native data is linked to the fact that native speakers tend to pronounce faster the common words belonging to their mother language, and thus the canonical pronunciations present in the native lexicon may not take into account these fast pronunciations, nor does the non-native variants. Moreover, the

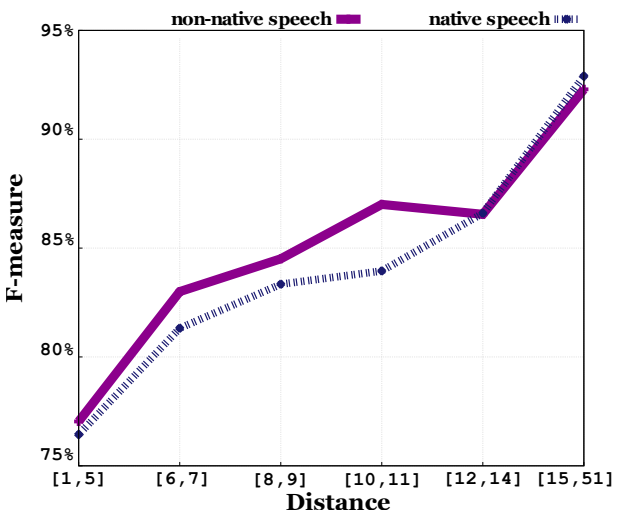

Fig. 4. Overall performance

fact that the acoustic models were trained on American English data (TIMIT corpus) may introduce some mismatch on native British English evaluation set.

\section{CONCLUSIONS}

This paper studied the rejection of incorrect entries, with a focus on non-native speech, in the context of foreign language learning.

Assessments carried out on two English corpora (one native and one non-native) have shown that it is important to train the decision functions on the same type of data (native training for tests on native speech, non-native training for tests on non-native speech). The use of alternative non-native pronunciations in the lexicon is necessary only for the task of non-native transcript verification. It is also useful to combine all comparison criteria (i.e. from both the phoneme loop and the word loop segmentations) through a logistic regression 


\begin{tabular}{|c|c|c|c|c|c|}
\hline Data & No. Syll & EER & FA & FR & F-measure \\
\hline \hline \multirow{5}{*}{ Native } & 3 & $21.83 \%$ & $20.72 \%$ & $22.51 \%$ & $78.4 \%$ \\
\cline { 2 - 6 } & 4 & $15.61 \%$ & $11.46 \%$ & $17.54 \%$ & $85.4 \%$ \\
\cline { 2 - 6 } & 5 & $14.23 \%$ & $9.39 \%$ & $17.13 \%$ & $86.6 \%$ \\
\cline { 2 - 6 } & 6 & $11.33 \%$ & $8.56 \%$ & $13.54 \%$ & $88.9 \%$ \\
\cline { 2 - 6 } & 7 & $9.39 \%$ & $6.08 \%$ & $10.77 \%$ & $91.5 \%$ \\
\cline { 2 - 6 } & all & $\mathbf{5 . 9 4 \%}$ & $\mathbf{8 . 0 1 \%}$ & $\mathbf{3 . 3 1 \%}$ & $\mathbf{9 4 . 3 \%}$ \\
\hline \multirow{5}{*}{ Non-native } & 3 & $20.05 \%$ & $16.20 \%$ & $21.85 \%$ & $80.9 \%$ \\
\cline { 2 - 6 } & 4 & $15.68 \%$ & $15.68 \%$ & $12.85 \%$ & $85.7 \%$ \\
\cline { 2 - 6 } & 5 & $12.85 \%$ & $13.11 \%$ & $11.05 \%$ & $87.9 \%$ \\
\cline { 2 - 6 } & 6 & $9.77 \%$ & $9.77 \%$ & $9.00 \%$ & $90.6 \%$ \\
\cline { 2 - 6 } & 7 & $8.48 \%$ & 8.48 & $8.23 \%$ & $91.6 \%$ \\
\cline { 2 - 6 } & all & $\mathbf{1 . 5 4 \%}$ & $\mathbf{2 . 0 6 \%}$ & $\mathbf{0 . 7 7 \%}$ & $\mathbf{9 8 . 6 \%}$ \\
\hline
\end{tabular}

Table 1. Results (EER (equal error rate), FA \& FR error rates plus their F-measure) on native data (native training, native lexicon, 10 comparison criteria) and on non-native data (non-native training, non-native lexicon, 10 comparison criteria)

function which assigns weights according to the importance of each criterion.

The optimal settings lead to reasonable false acceptance and false rejection error-rates $(2.06 \%$ and $0.77 \%$ for nonnative data, $8.01 \%$ and $3.31 \%$ for native data) when the pronounced sentences are entirely different from the expected sentences. For partially different sentences, starting from a distance of 6 phoneme changes we can obtain a performance greater than $80 \%$.

\section{REFERENCES}

[1] D. Herron, W. Menzel, E. Atwell, R. Bisiani, F. Daneluzzi, R. Morton, and J. A. Schmidt, "Automatic localization and diagnosis of pronunciation errors for second-language learners of english.," in $E U$ ROSPEECH, 1999.

[2] S.M Witt and S.J Young, "Phone-level pronunciation scoring and assessment for interactive language learning," Speech Communication, vol. 30, pp. 95 - 108, 2000.

[3] M. Eskenazi, Y. Ke, J. Albornoz, and K. Probst, "The fluency pronunciation trainer: Update and user issues," in Proceedings INSTiL2000, 2000.

[4] G. Henry, A. Bonneau, and V. Colotte, "Tools devoted to the acquisition of the prosody of a foreign language," in International Congress of Phonetic Sciences, 2007, pp. $1593-1596$.

[5] L. Mesbahi, D. Jouvet, A. Bonneau, D. Fohr, I. Illina, and Y. Laprie, "Reliability of non-native speech automatic segmentation for prosodic feedback," in Workshop on Speech and Language Technology in Education. ISCA, 2011.

[6] D. Jouvet, L. Mesbahi, A. Bonneau, D. Fohr, I. Illina, and Y. Laprie, "Impact of pronunciation variant fre- quency on automatic non-native speech segmentation," in Language and Technology Conference, 2011, pp. 145 -148 .

[7] Issam Bazzi and James R. Glass, "Modeling out-ofvocabulary words for robust speech recognition," in $I C$ SLP, 2000, vol. 1, pp. $401-404$.

[8] C. White, G. Zweig, L. Burget, P. Schwarz, and H. Hermansky, "Confidence estimation, oov detection and language id using phone-to-word transduction and phonelevel alignments," ICASSP, pp. 4085-4088, 2008.

[9] R. Boite, Traitement de la parole, Presses polytechniques et universitaires romandes, 2000.

[10] M.H. Davel, C.J. van Heerden, and E. Barnard, "Validating smartphone-collected speech corpora," SLTU, 2012.

[11] S. Dreiseitl and L. Ohno-Machado, "Logistic regression and artificial neural network classification models," Journal of Biomedical Informatics, vol. 35, pp. 352 359, 2002.

[12] M. Dargnat, A. Bonneau, and V. Colotte, "Perception et apprentissage des contours prosodiques en 11 et en 12," "http://mathilde.dargnat.free.fr/INTONALE/intonaleweb.html", 2010.

[13] S. Young, G. Evermann, D. Kershaw, G. Moore, J. Odell, D. Ollason, D. Povey, V. Valtchev, and P. Woodland, The HTK Book (for HTK version 3.2), Cambridge University Engineering Department, 2002.

[14] J. Garofolo, "An acoustic phonetic continuous speech database," Speech communication, vol. 30, pp. 95 - 198, 2000.

[15] S. Galliano, G. Gravier, and L. Chaubard, "The ester2 evaluation campaign for the rich transcription of french radio broadcasts," Interspeech, 2009.

[16] Andrew Hunt, ," "http://www.speech.cs.cmu.edu/cgibin/cmudict", 1996. 